\title{
Erratum
}

\section{Erratum to "Seminormal Structure and Fixed Points of Cyclic Relatively Nonexpansive Mappings"}

\author{
Moosa Gabeleh ${ }^{1}$ and Naseer Shahzad ${ }^{2}$ \\ ${ }^{1}$ Department of Mathematics, Ayatollah Boroujerdi University, Boroujerd, Iran \\ ${ }^{2}$ Department of Mathematics, King Abdulaziz University, P.O. Box 80203, Jeddah 21589, Saudi Arabia \\ Correspondence should be addressed to Naseer Shahzad; nshahzad@kau.edu.sa \\ Received 11 May 2014; Accepted 5 June 2014; Published 8 July 2014 \\ Copyright (C) 2014 M. Gabeleh and N. Shahzad. This is an open access article distributed under the Creative Commons Attribution \\ License, which permits unrestricted use, distribution, and reproduction in any medium, provided the original work is properly \\ cited.
}

In this paper we point out some corrections needed in [1].

Recently, a geometric notion of seminormal structure has been introduced as follows.

Definition 1 (see [1]). A convex pair $(A, B)$ in a Banach space $X$ is said to have seminormal structure if, for any bounded, closed, and convex pair $\left(K_{1}, K_{2}\right) \subseteq(A, B)$ with $\delta\left(K_{1}, K_{2}\right)>0$, there exits $(p, q) \in K_{1} \times K_{2}$ such that

$$
\max \left\{\delta_{p}\left(K_{2}\right), \delta_{q}\left(K_{1}\right)\right\}<\delta\left(K_{1}, K_{2}\right)
$$

It has been remarked in [1] that the pair $(A, A)$ has seminormal structure if and only if $A$ has normal structure in the sense of Brodskil and Mil'man [2]. We revise this remark as follows. If the pair $(A, A)$ has seminormal structure, then $A$ has normal structure in the sense of Brodskii and Mil'man. Indeed, if the set $A$ has normal structure, then $(A, A)$ may not have seminormal structure. We illustrate this with the following example.

Example 2. Let $X:=\mathbb{R}$ with the usual metric and let $A:=$ $[0,1]$. Then $A$ has normal structure because $A$ is a nonempty, bounded, closed, and convex subset of the uniformly convex Banach space $X$. Suppose $K_{1}:=\{0\}$ and $K_{2}:=\{1\}$. Then $\max \left\{\delta_{p}\left(K_{2}\right), \delta_{q}\left(K_{1}\right)\right\}=\delta\left(K_{1}, K_{2}\right)$; that is, $(A, A)$ does not have seminormal structure.

The following notion has also been given in [1].

Definition 3 (see [1]). A nonempty, bounded, closed, and convex pair $(A, B)$ of a normed linear space is said to have property (D) provided that for each nonempty, closed, and convex pair $(E, F) \subseteq(A, B)$ one has

$$
\min \{\operatorname{diam}(E), \operatorname{diam}(F)\} \leq \delta(E, F) .
$$

In [1], the following proposition has been obtained to derive Corollary 5 (see Corollary 12 in [1]).

Proposition 4 (see Proposition 11 in [1]). Let $(A, B)$ be a nonempty, bounded, closed, and convex pair in a uniformly convex Banach space $X$ such that $(A, B)$ has the property $(D)$. Then $(A, B)$ has seminormal structure.

Corollary 5 (see Corollary 12 in [1]). Let $(A, B)$ be a nonempty, bounded, closed, and convex pair in a uniformly convex Banach space $X$ such that $(A, B)$ has the property $(D)$. Assume that $T: A \cup B \rightarrow A \cup B$ is a cyclic relatively nonexpansive mapping. Then $T$ has a fixed point.

In the following, we give a counterexample to Proposition 4 which suggests that the result of Corollary 5 should be revised.

Example 6. Let $X:=\mathbb{R}$ with the usual metric and let $A:=$ $[0,1]$ and $B:=[2,3]$. It is clear that $(A, B)$ has the property (D). Now, consider $K_{1}:=\{0\}$ and $K_{2}:=\{3\}$ and suppose $(p, q)=(0,3)$. Then

$$
\max \left\{\delta_{p}\left(K_{2}\right), \delta_{q}\left(K_{1}\right)\right\}=\delta\left(K_{1}, K_{2}\right) ;
$$

that is, $(A, B)$ does not have seminormal structure. 
Using an argument similar to that in the proof of Proposition 11 in [1], we are able to correct Corollary 5 as follows.

Corollary 7. Let $(A, B)$ be a nonempty, bounded, closed, and convex pair in a uniformly convex Banach space $X$ such that $(A, B)$ has the property (D). If $T: A \cup B \rightarrow A \cup B$ is a cyclic relatively nonexpansive mapping, then either $A \cap B$ is nonempty and $T$ has a fixed point in $A \cap B$ or $T$ has a best proximity point.

Proof. Suppose $\mathscr{F}$ denotes the collection of all nonempty, closed, and convex pairs $(E, F) \subseteq(A, B)$ such that $T$ is cyclic on $E \cup F$ and there exists a pair $(p, q) \in E \times F$ for which $\|p-q\|=\operatorname{dist}(A, B)$. Note that $\left(A_{0}, B_{0}\right) \in \mathscr{F}$. By using Zorn's lemma we can see that $\mathscr{F}$ has a minimal element, say $\left(K_{1}, K_{2}\right)$. If $\delta\left(K_{1}, K_{2}\right)=0$, then $A \cap B$ is a nonempty, bounded, closed, and convex subset of a uniformly convex Banach space $X$ and $T: A \cap B \rightarrow A \cap B$ is a nonexpansive mapping. Thus $T$ has a fixed point and we are finished. So, we assume that $\delta\left(K_{1}, K_{2}\right)>0$. We now consider the following cases.

Case 1. If $\min \left\{\operatorname{diam}\left(K_{1}\right), \operatorname{diam}\left(K_{2}\right)\right\}=0$, we may assume that $K_{1}=\left\{x^{*}\right\}$. Consequently, there exists $y^{*} \in K_{2}$ such that $\| x^{*}-$ $y^{*} \|=\operatorname{dist}(A, B)$. Since $T$ is a cyclic relatively nonexpansive mapping, we have

$$
\left\|x^{*}-T x^{*}\right\|=\left\|T y^{*}-T x^{*}\right\| \leq\left\|y^{*}-x^{*}\right\|=\operatorname{dist}(A, B) .
$$

This implies that $T$ has a best proximity point.

Case 2. If $\min \left\{\operatorname{diam}\left(K_{1}\right), \operatorname{diam}\left(K_{2}\right)\right\}>0$, by an argument similar to that in Proposition 11 of [1], we conclude that there exists a pair $(p, q) \in K_{1} \times K_{2}$ such that $\max \left\{\delta_{p}\left(K_{2}\right), \delta_{q}\left(K_{1}\right)\right\}<$ $\delta\left(K_{1}, K_{2}\right)$. By analogous proof of Theorem 8 in [1], we obtain that $\delta\left(K_{1}, K_{2}\right)=0$, which is a contradiction.

\section{References}

[1] M. Gabeleh and N. Shahzad, "Seminormal structure and fixed points of cyclic relatively nonexpansive mappings," Abstract and Applied Analysis, vol. 2014, Article ID 123613, 8 pages, 2014.

[2] M. S. Brodskiĭ and D. P. Mil'man, "On the center of a convex set," Doklady Akademii Nauk SSSR, vol. 59, pp. 837-840, 1948 (Russian). 


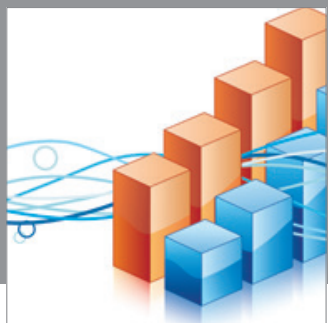

Advances in

Operations Research

mansans

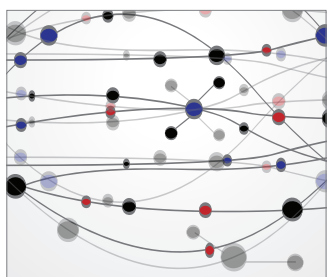

The Scientific World Journal
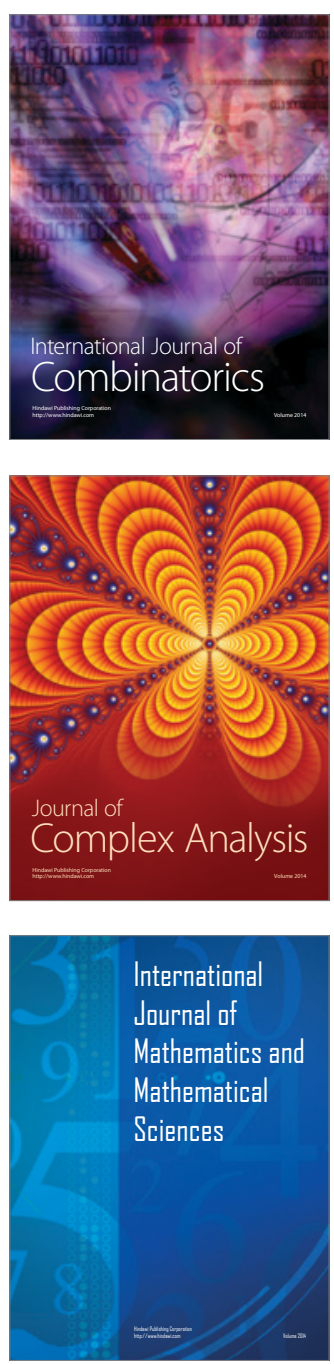
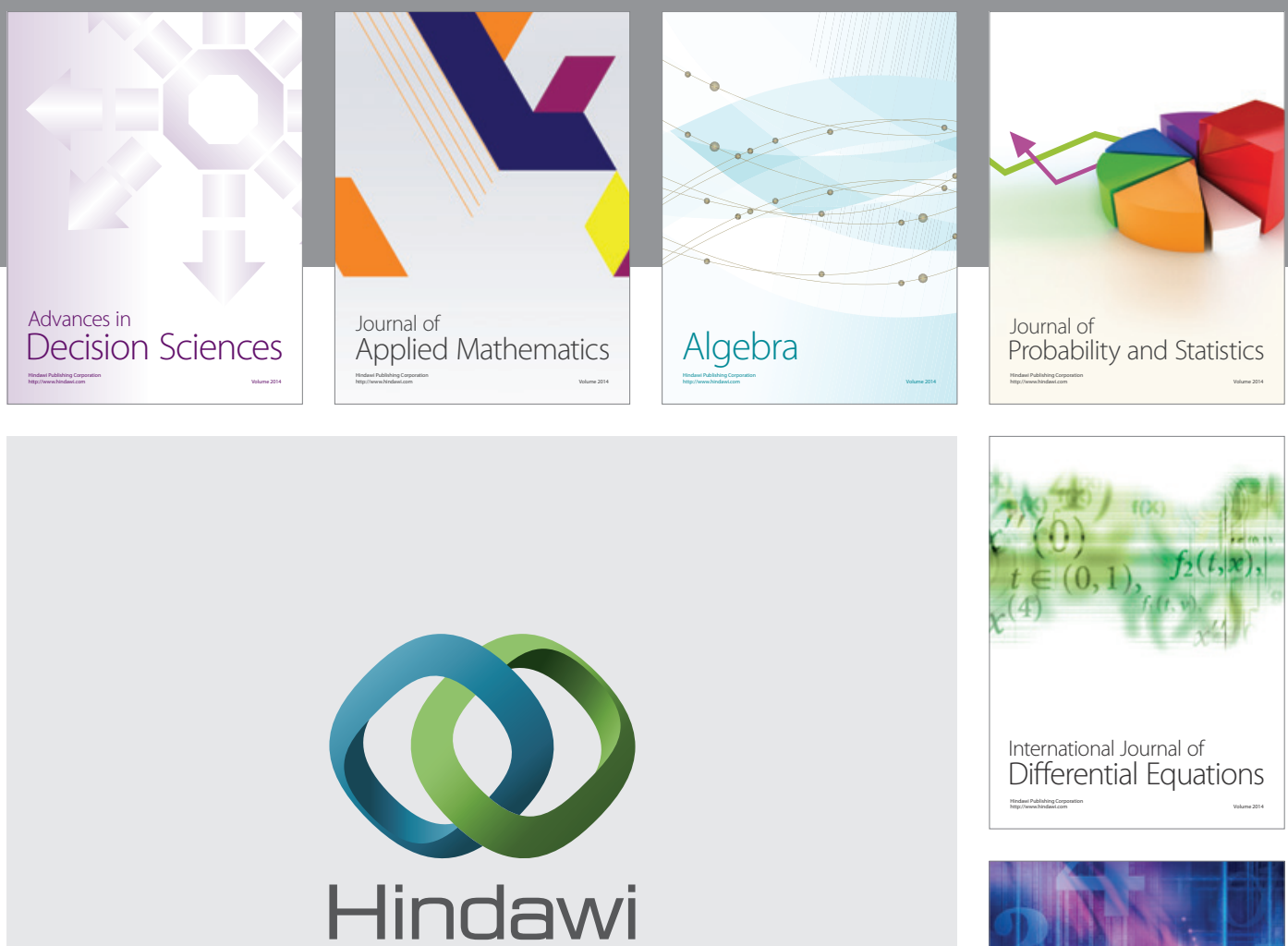

Submit your manuscripts at http://www.hindawi.com
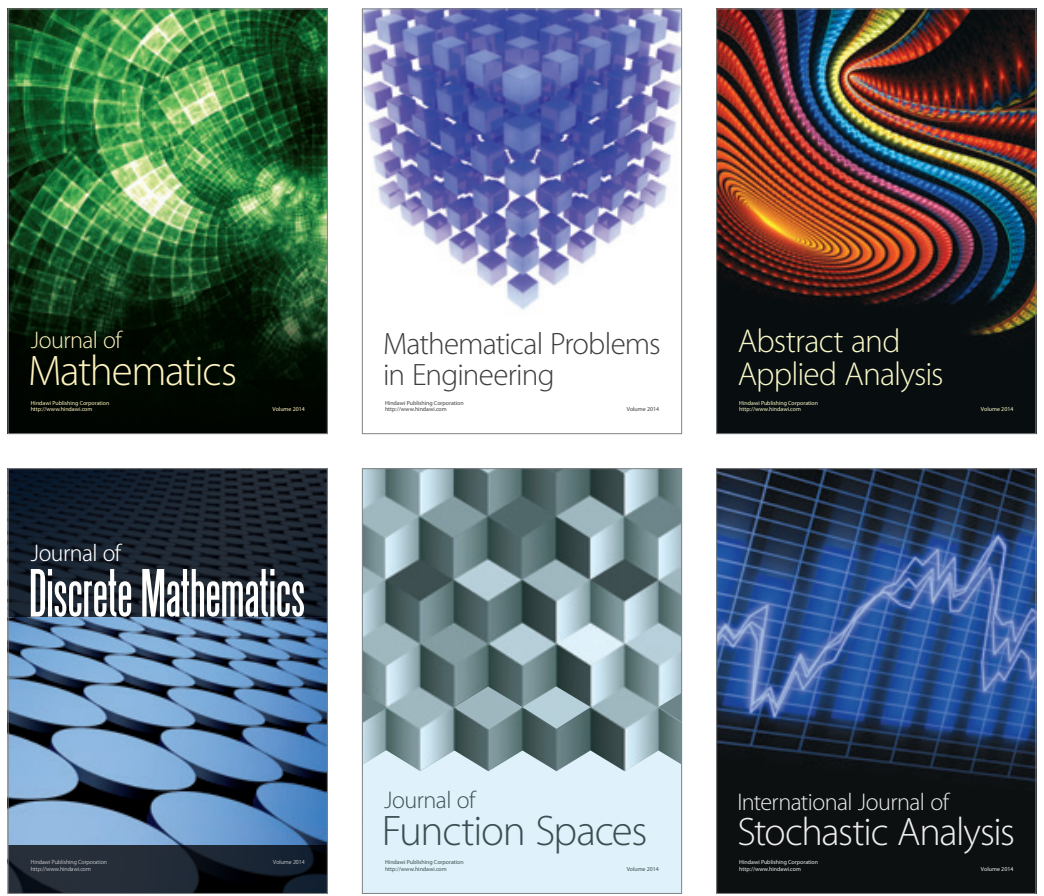

Journal of

Function Spaces

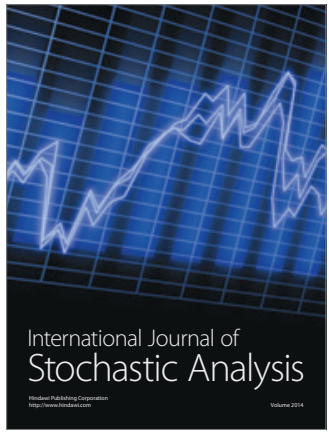

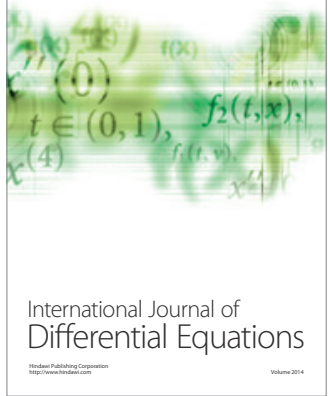
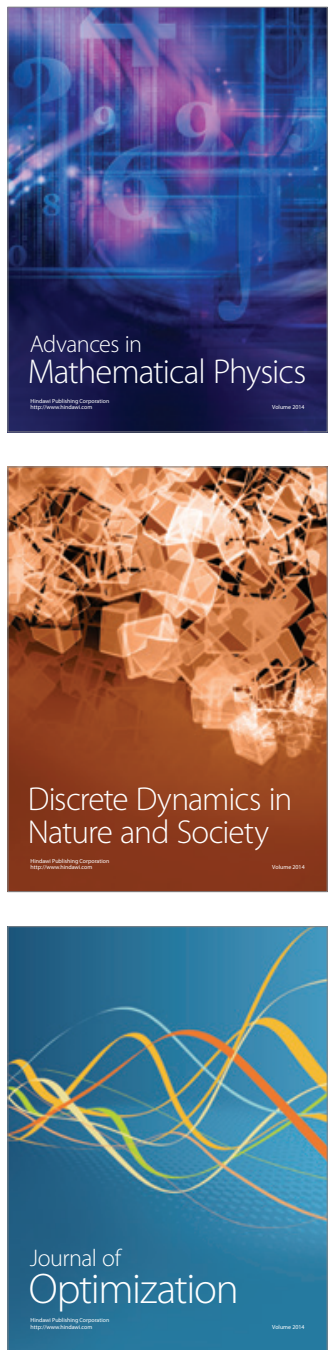